\title{
A Strong Robust Zero-Watermarking Scheme Based on Shearlets' High Ability for Capturing Directional Features
}

\author{
Jian Zhao, Wensheng Xu, Shunli Zhang, Shuaishuai Fan, and Wanru Zhang \\ School of Information Science and Technology, Northwest University, Xian 710069, China \\ Correspondence should be addressed to Jian Zhao; zjctec@nwu.edu.cn
}

Received 14 April 2016; Revised 29 September 2016; Accepted 10 October 2016

Academic Editor: Jinyang Liang

Copyright ( 2016 Jian Zhao et al. This is an open access article distributed under the Creative Commons Attribution License, which permits unrestricted use, distribution, and reproduction in any medium, provided the original work is properly cited.

\begin{abstract}
Zero-watermarking is a blind digital watermarking method. It has reached the point where the robustness and the imperceptibility can arrive at a good balance. In this paper, a strong robust zero-watermarking scheme is proposed which employs multiresolution and multiscale representation characteristics of nonsubsampled shearlet transform to analyze the direction features of the given image. The effectiveness of the proposed scheme for dealing with many kinds of attack such as compression, noise addition, and scaling is demonstrated by the experimental results. When compared with other zero-watermarking schemes using counterpart transforms like discrete wavelet transform, the experimental results show that the proposed watermarking scheme can get better performance.
\end{abstract}

\section{Introduction}

Digital watermarking techniques [1-4] are effective means to protect copyright of the digital media. It has attracted a lot of attention in the last decade. However, traditional digital watermarking system which modifies the host image to some extent has been studied for a long time. There still remain such problems about watermarks as robustness, imperceptibility, or unavailability of the host image in the extracting process. A zero-watermarking system is a type of watermarking schemes that is encrypted with the host image [5] instead of hiding information inside the host image. It can be a good alternative watermarking method for its bringing no distortion to the host image. Thus, the quality of the host image can be ensured.

The crucial step in the zero-watermarking system is to extract stable internal feature information from the host image. As a higher dimensional signal, images are governed by anisotropic directional features [6], such as edges or textures. How to efficiently represent images with rich direction feature information is a central problem in image processing fields. The wavelet transform is well known for capturing the geometry of image edges because it provides a good multiresolution representation of one-dimensional piecewise smooth signals $[7,8]$, but it is restricted by the limited directionality in its filtering structures.

These disappointing behaviors indicate that more powerful representation methods are needed. Thus, numerous approaches for efficiently representing direction features in images have been proposed, including directional filter banks [9], Contourlet [10-12], and shearlets [6, 10, 13, 14]. Among all these representation systems, shearlets exhibit many outstanding properties: the potential ability to use the power of multiscale to capture geometry of multidimensional data, optimally sparse approximation of anisotropic features. It is an affine system containing a single mother function that is parameterized by scaling, shearing, and translating parameters [15]. It is argued that shearlets can capture direction features more precisely and efficiently $[13,15]$. All these brilliant advantages make shearlets the most successful and versatile representation system.

Zero-watermark algorithm was proposed by Wen et al. [16], which does not change the original image. And the most important step is to construct a zero-watermark with the essential characteristics of the host image. In previous literatures [17], though NSST was applied in zero-watermarking algorithms. It was not clearly stated what kind of essential parts of the images should be considered and why NSST is a 


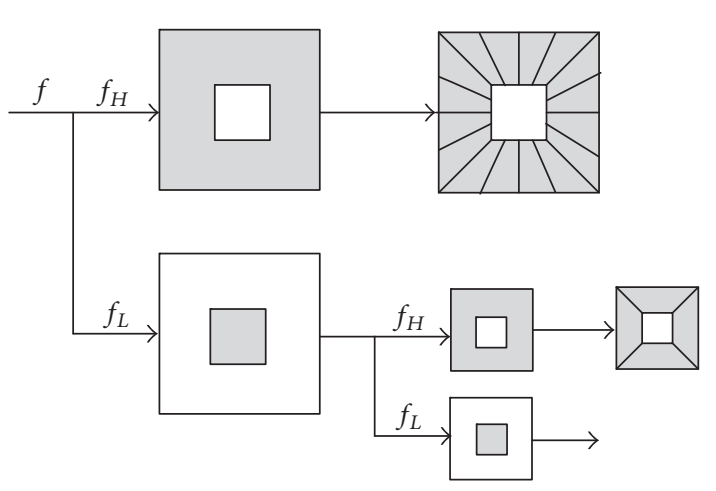

(a)

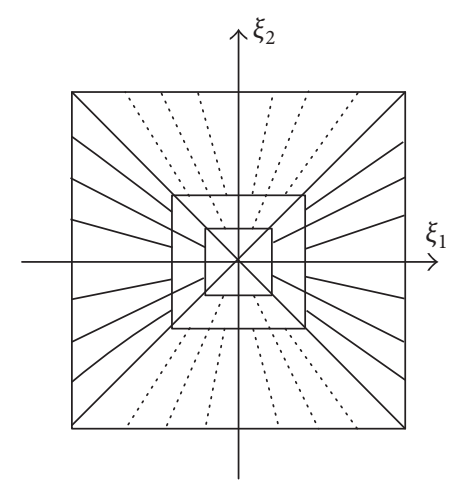

(b)

Figure 1: (a) The illustration of the succession of LP decomposition and shearlet directional filtering. (b) The tiling of shearlets in the frequency domain.

better tool for representing images. Based on former studies [18], a novel zero-watermarking scheme is proposed through using shearlets and matrix norm to analyze image direction features and transform domain coefficients respectively. In this paper, image directionality is taken into account as a kind of essential property. And direction information intensity is defined to quantify the measurements. The experimental results demonstrate that the proposed algorithm can get remarkable robust ability. The comparison with latest methods proves that the zero-watermarking scheme that use shearlets is superior to those based on DWT.

\section{Theoretical Background}

2.1. Basic Theory of Shearlets [6, 13, 14, 19]. 2-dimensional affine system with composite dilations is as follows:

$$
\begin{aligned}
& S_{A B}(\psi)=\left\{\psi_{j, l, k}(x)=|\operatorname{det} A|^{j / 2} \psi\left(B^{l} A^{j} s-k\right): j, l\right. \\
& \left.\quad \in Z, k \in Z^{2}\right\} .
\end{aligned}
$$

If $S_{A, B}(\psi)$ forms a Parseval frame, the elements of this system are called composite wavelets. Shearlets are a special example of composite wavelets in $L^{2}\left(R^{2}\right)$. There are collections of the form (1) where $A$ is the anisotropic dilation matrix and $B$ is the shear matrix:

$$
\begin{aligned}
& A=\left(\begin{array}{ll}
4 & 0 \\
0 & 2
\end{array}\right), \\
& B=\left(\begin{array}{ll}
1 & 1 \\
0 & 1
\end{array}\right) ;
\end{aligned}
$$

for any $\xi=\left(\xi_{1}, \xi_{2}\right), \xi_{1} \neq 0$, let

$$
\widehat{\psi}^{(0)}(\xi)=\widehat{\psi}^{(0)}\left(\xi_{1}, \xi_{2}\right)=\widehat{\psi}_{1}\left(\xi_{1}\right) \widehat{\psi}_{2}\left(\frac{\xi_{2}}{\xi_{1}}\right)
$$

where $\widehat{\psi}_{1}, \widehat{\psi}_{2} \in C^{\infty}(\widehat{R}) \operatorname{supp} \widehat{\psi}_{1} \subset[-1 / 2,-1 / 16] \cup[1 / 16$, $1 / 2] \operatorname{supp} \widehat{\psi}_{2} \subset[-1,1]$ assume that

$$
\sum_{j \geq 0}\left|\widehat{\psi}_{1}\left(2^{-2 j} \omega\right)\right|^{2}=1, \quad|\omega| \geq \frac{1}{8}
$$

for $j \geq 0$,

$$
\sum_{l=-2^{j}}^{2^{j}-1}\left|\widehat{\psi}_{2}\left(2^{j} \omega-l\right)\right|^{2}=1, \quad|\omega| \leq 1
$$

from the supporting condition $\widehat{\psi}_{1}$ and $\widehat{\psi}_{2}, \psi_{j, l, k}$ in frequency domain is supported with

$$
\begin{gathered}
\widehat{\psi}_{j, l, k}^{(0)} \subset\left\{\left(\xi_{1}, \xi_{2}\right): \xi_{1} \in\left[-2^{2 j-1},-2^{2 l-4}\right]\right. \\
\left.\cup\left[2^{2 j-4}, 2^{2 j-1}\right],\left|\frac{\xi_{2}}{\xi_{1}}+l 2^{-j}\right| \leq 2^{-j}\right\}
\end{gathered}
$$

That is, each element $\widehat{\psi}_{j, l, k}(x)$ is supported on a pair of trapezoids, of approximate size $2^{2 j} \times 2^{j}$, oriented along lines of the slope $l 2^{-j}$.

Nonsubsampled shearlet transform (NSST) is implemented by two steps. Firstly, apply Laplace Pyramid scheme to decompose the host image $f$ into a low-pass image $f_{L}$ and several different scale levels, namely, high-pass component $f_{H}$. Secondly, the high-pass part is decomposed by discrete shearlet transform to get direction subbands. Because the image is decomposed without either downsampling or upsampling, the outputs have the same size as the original image. Figure 1(a) shows the illustration of LP decomposition succession and shearlet directional filtering. Figure 1(b) shows the tilling of frequency plane of shearlets.

2.2. Matrix Norm. If $A$ is a nonnegative matrix, its singular value decomposition (SVD) is defined as follows:

$$
\begin{aligned}
A & =U S V^{T}, \\
U^{T} A V & =S=\operatorname{diag}\left(\sigma_{1}, \sigma_{2}, \sigma_{3}, \ldots, \sigma_{p}\right),
\end{aligned}
$$

where $p$ is minimum of $n$ and $m, \sigma_{i}$ is the singular value of matrix $A, \sigma_{1}>\sigma_{2}>\sigma_{3} \geq \cdots \geq \sigma_{p}$, $U$ is left singular value 


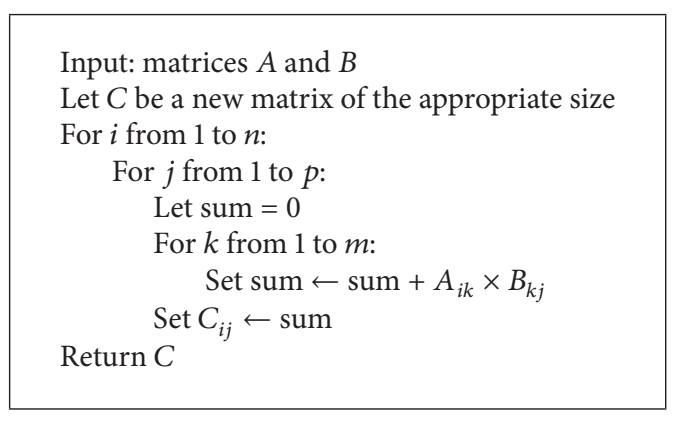

Algorithm 1:

vector, and $V$ is right singular value vector. Spectrum norm and $F$-norm of nonnegative matrix $A$ are defined as follows:

$$
\begin{aligned}
& \|A\|_{2}=\sqrt{\lambda_{\max }\left(A^{T} A\right)}=\sigma_{1}, \\
& \|A\|_{F}=\sqrt{\sum_{i=1}^{m} \sum_{j=1}^{n} a_{i j}^{2}}=\sum_{i=1}^{p} \lambda_{i}=\sigma_{1}^{2}+\sigma_{2}^{2}+\cdots+\sigma_{p}^{2} .
\end{aligned}
$$

Matrix's spectrum norm is maximal singular; $F$-norm is the square sum of all singular values. From formula (8), when $F=2$, the matrix 2-norm is equivalent to the largest singular value.

SVD is widely applied to digital watermarking technologies $[20,21]$ and it effectively reveals that the larger singular values of an image do not change significantly when common image processing attacks are performed on this image. However, SVD calculating will increase algorithm's complexity and computing cost, since the SVD process has matrix product. The definition of matrix multiplication is that if $C=A B$ for an $n \times m$ matrix $A$ and an $m \times p$ matrix $B$, then $C$ is an $n \times p$ matrix with entries.

From this, a simple algorithm can be constructed which loops over the indices $i$ from 1 through $n$ and $j$ from 1 through $p$, computing the above using a nested loop in Algorithm 1.

This algorithm takes time $O(n m p)$. A common simplification for the purpose of the algorithm analysis is to assume that the inputs are all square matrices of size $n \times n$, in which case the running time is $O\left(n^{3}\right)$. The intimate relationship between matrix 2 norm and singular value is that matrix 2norm is equivalent to the largest matrix singular value. Here we compared the calculating costs of SVD and matrix 2norm. Table 1 shows the time costs when calculating SVD and 2-norm of a matrix by Matlab, R2013b. The CPU is Intel ${ }^{\circledR}$ Core $^{\mathrm{TM}}$ 2, $3.00 \mathrm{GHz}, 32$ bit.

From Table 1, calculating 2-norms is faster than calculating SVD. When matrixes size become larger, like Lena $(256 \times 256)$, the advantage of using 2-norm instead of SVD to acquire largest singular value becomes much more explicit. For the sake of time consuming, therefore, in this paper, matrix 2-norm is used instead of singular values to zero-watermarking embedding because of the intimate relationship between matrix norm and singular values.
TABLE 1: Time costs comparison when calculating SVD and 2-norm of different matrix.

\begin{tabular}{lcc}
\hline Size of matrix & SVD $(\mathrm{s})$ & 2-norm $(\mathrm{s})$ \\
\hline Lena $(128 \times 128)$ & 0.0083 & 0.0030 \\
Lena $(256 \times 256)$ & 0.4043 & 0.1051 \\
Man $(128 \times 128)$ & 0.0071 & 0.0054 \\
Man $(256 \times 256)$ & 0.2474 & 0.1022 \\
Plane $(128 \times 128)$ & 0.0084 & 0.0039 \\
Plane $(256 \times 256)$ & 0.0508 & 0.0298 \\
\hline
\end{tabular}

\section{A Novel Zero-Watermarking Scheme}

3.1. Image Multidirection Features. There exist many kinds of directional components in the host image like textures or edges. A large number of literatures $[22,23]$ talked about methods to portray texture and edge directionality. Inspired by this special characteristic, in the proposed algorithm, multidirectionality of an image is regarded as a stable feature that should be given more attention in digital watermarking techniques.

Since the direction feature of an image is diverse, however, it is undisputable that only a few directions that DWT or DCT provides may not be sufficient enough to represent the direction information of an image [24]. Thus, high direction sensitivity becomes the most important property of the tool selected for image direction analysis. According to the discussion of shearlets in Section 2, NSST is suitable for dealing with this task because of its direction sensitivity and sparse approximation of anisotropic features. Figure 2 illustrates the two-level NSST of the Zoneplate image. The first scale-level is decomposed into 4 directional subbands, and the second scale-level is decomposed into 8-directional subbands. In the following, NSST is used to illustrate the method to extract direction feature information.

Let artificial image (a) in Figure 3 be denoted as $I$, and then perform the forward NSST to obtain multidirection and multiscale representation:

$$
S H=\operatorname{NSST}^{+}(I),
$$

where $\mathrm{NSST}^{+}$denotes forward NSST and $\mathrm{SH}=\left\{\mathrm{SH}_{s, d}\right\}$ denotes the NSST coefficient matrix set of $I$ at scale $s$, direction $d$. As is shown in Figure 3, when single direction coefficients are reconstructed to the time domain, specific image direction features can be seen clearly. However, as is shown in Figure 4, there are many kinds of direction components existing in real images, such as geometrical lines, edges, and textures. Direction information intensity here is adopted to measure how much feature information the subband has in given direction. Direction information intensity is defined by the following rule:

$$
\begin{aligned}
S_{s, d} & =\operatorname{NSST}^{-}\left(S H_{s, d}\right), \\
A_{d} & =\sum_{i=1}^{M} \sum_{j=1}^{N}\left|S_{s, d}(i, j)\right|,
\end{aligned}
$$



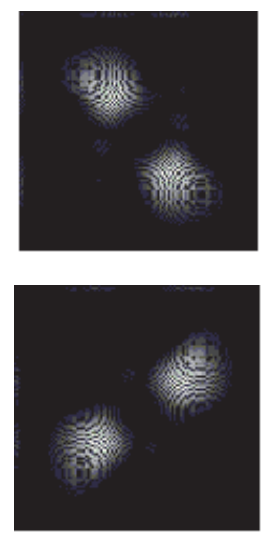

(c)
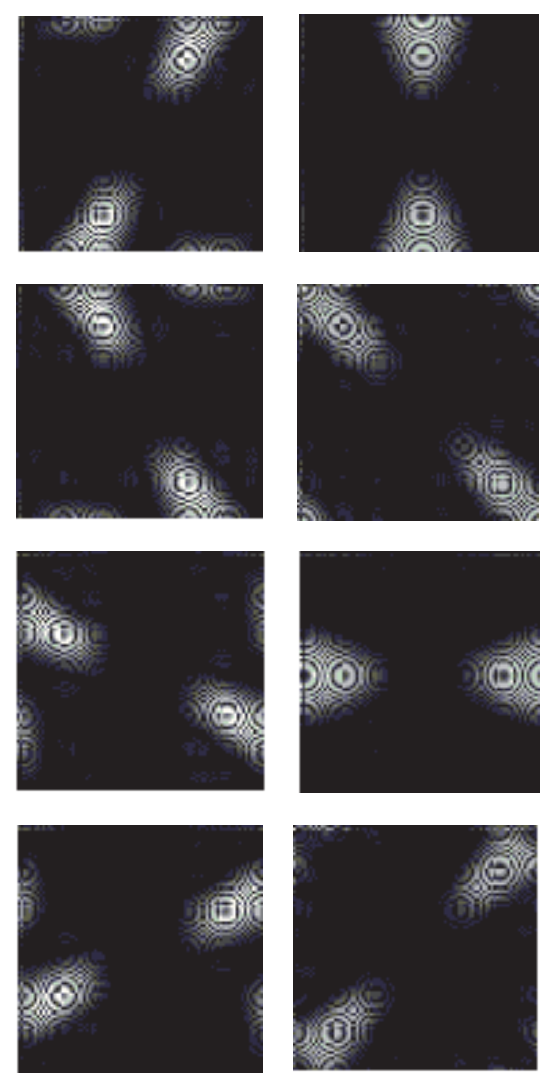

(d)

FIGURE 2: An illustration of NSST. (a) Original image (b) low-pass coefficients. Image of the detail shear coefficients are shown in (c) and (d) with 4 and 8 directions, respectively, corresponding to the different directional bands illustrated in Figure 1(a).

TABLE 2: The eight direction feature information intensity values of image Lena.

\begin{tabular}{ccccccccc}
\hline & $A_{1}$ & $A_{2}$ & $A_{3}$ & $A_{4}$ & $A_{5}$ & $A_{6}$ & $A_{7}$ & $A_{8}$ \\
\hline Values $\left({ }^{*} \mathrm{E} 05\right)$ & 1.24 & 1.37 & 1.38 & 1.45 & 1.59 & 2.31 & 2.08 & 1.36 \\
\hline
\end{tabular}

where $\mathrm{NSST}^{-}$denotes inverse NSST, $S_{s . d}$ indicates the direction coefficients in the time domain. The larger the $A_{d}$ is, the richer the direction information the subband has at scale $s$, direction $d$.

This direction feature information can be used for selecting watermark embedding position. To obtain the robustness, the directional subband with the largest direction feature information intensity will be selected to construct zerowatermark. Table 2 shows direction feature information intensity values $A_{d}$ of image Lena.

3.2. Proposed Algorithm. Based on the former analysis, a novel zero-watermarking scheme is proposed in this section. The actual watermark is a binary image denoted as $W=$ $\{w(i, j)=0 / 1,1 \leq i \leq m, 1 \leq j \leq n\}$. The host image is a gray-level image denoted as $F=\{f(i, j), 1 \leq i \leq M, 1 \leq j \leq$ $N\}$. Without the loss of generality, the size of host image and watermark must satisfy $M / n=p, N / n=q p, q$ are integers.
In this paper, the size of watermark is $32 \times 32$, the size of the host images is $512 \times 512$. That is, $p=q=16$.

\section{(1) Watermark Embedding}

Step 1. Apply forward NSST to decompose the host image $F$ to gain multiscale and multiresolution representation.

Step 2. Reconstruct coefficients of 8 direction subbands to the time domain. All these 8-direction subbands are marked from $d_{1}$ to $d_{8}$.

Step 3. Calculate direction feature information intensity of each subband according to formula (9) to determine embedding position $d_{i}$.

Step 4. Divide the selected subband into nonoverlapping $m \times n$ blocks followed by calculating 2-norms of each block and the largest 2 -norm is $a$; the minimum 2-norm is $b$. Then, compare all these 2-norms with the threshold $t$ defined as below:

$$
t=\frac{a+b}{2}
$$

When the 2-norm is larger than $t, K(i, j)=1$; otherwise, $K(i, j)=0$. 


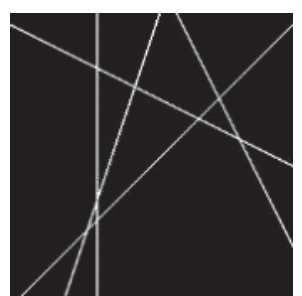

(a)

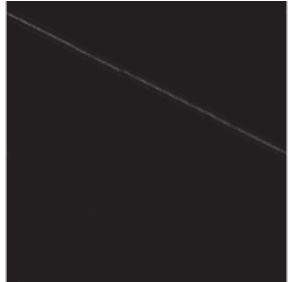

$S_{2,1}$

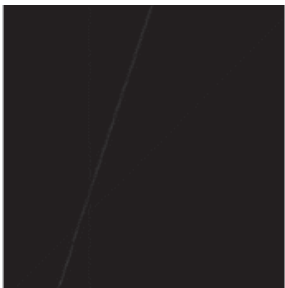

$S_{2,5}$

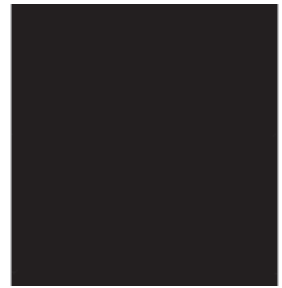

$S_{2,2}$

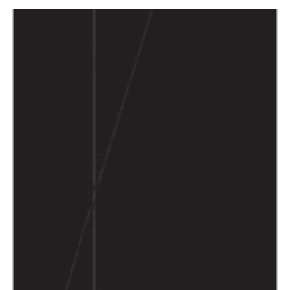

$S_{2,6}$

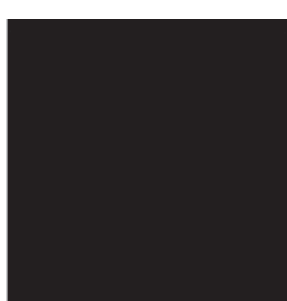

$S_{2,3}$

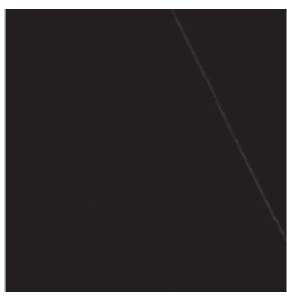

$S_{2,7}$

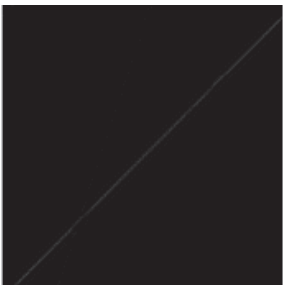

$S_{2,4}$

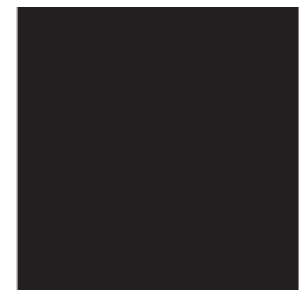

$S_{2,8}$

FIGURE 3: Eight direction components extracted by inverse NSST at $s=2, d=1,2, \ldots, 8$.

Step 5. The zero-watermarking $Z W$ is obtained from the binary embedding key $K$ and the actual watermarking $W$ based on the exclusive-or (XOR) operation:

$$
Z W=\operatorname{XOR}(K, W) .
$$

At last, $Z W$ can be used to assert the copyright of the image when needed.

\section{(2) Watermark Extracting}

Step 1. Apply forward NSST to decompose the tested image $F^{\prime}$.

Step 2. The extracting position and embedding position must be the same; thus, the extracting position is also $d_{i}$.

Step 3. Divide the selected subband into nonoverlapping $m \times n$ blocks followed by calculating 2-norms of each block and the largest 2-norm is $a^{\prime}$, the minimum 2-norm is $b^{\prime}$. Then, compare 2-norms with the threshold $t^{\prime}$ defined below like (12). When the 2-norm is larger than $t^{\prime} K^{\prime}(i, j)=1, K^{\prime}(i, j)=$ 0 :

$$
t^{\prime}=\frac{a^{\prime}+b^{\prime}}{2}
$$

Step 4. The extracted watermark $W^{\prime}$ can be obtained based on the following XOR operation

$$
W^{\prime}=\operatorname{XOR}\left(Z W, K^{\prime}\right) \text {. }
$$

\section{Performance Evaluation and Analysis}

To verify the effectiveness of the proposed algorithm, a series of experiments were conducted using images from USISIPI image database. Here six $512 \times 512$ sized grayscale images, which are shown in Figure 5, are taken as host images to quantify analysis of the proposed watermarking scheme. Structural similarity (SSIM) [25], which is used to measure the perceived quality of two images, and normalized correlation (NC), which is used to measure the resemblance between extracted and actual watermark, are applied. The SSIM index is calculated on various windows of an image. The measure between two windows $x$ and $y$ of common size $N \times N$ is

$$
\operatorname{SSIM}(x, y)=\frac{\left(2 \mu_{x} \mu_{y}+C_{1}\right)\left(2 \sigma_{x y}+C_{2}\right)}{\left(\mu_{x}^{2}+\mu_{y}^{2}+C_{1}\right)\left(\sigma_{x}^{2}+\sigma_{y}^{2}+C_{2}\right)},
$$

where $\mu_{x}$ and $\mu_{y}$ are the averages of $x$ and $y, \sigma_{x}^{2}$ and $\sigma_{y}^{2}$ are the variances of $x$ and $y$, and $\sigma_{x y}$ is the covariance of $x$ and $y . C_{1}$ and $C_{2}$ are constants, where $C_{1}=\left(k_{1} L\right)^{2}, C_{2}=\left(k_{2} L\right)^{2}, k_{1}=$ $0.01, k_{2}=0.03$ by default, and $L$ is the dynamic range of the pixel-values.

$\mathrm{NC}$ is calculated as

$$
\mathrm{NC}=\frac{\sum_{i=1}^{m} \sum_{j=1}^{n} W(i, j) * W^{\prime}(i, j)}{\sqrt{\sum_{i=1}^{m} \sum_{j=1}^{n}[W(i, j)]^{2}} \sqrt{\sum_{i=1}^{m} \sum_{j=1}^{n}\left[W^{\prime}(i, j)\right]^{2}}},
$$




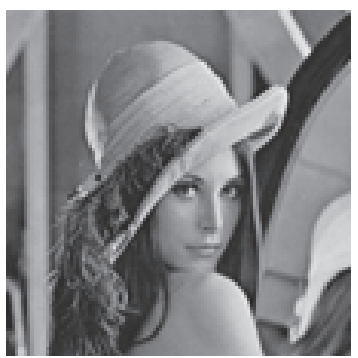

(a)
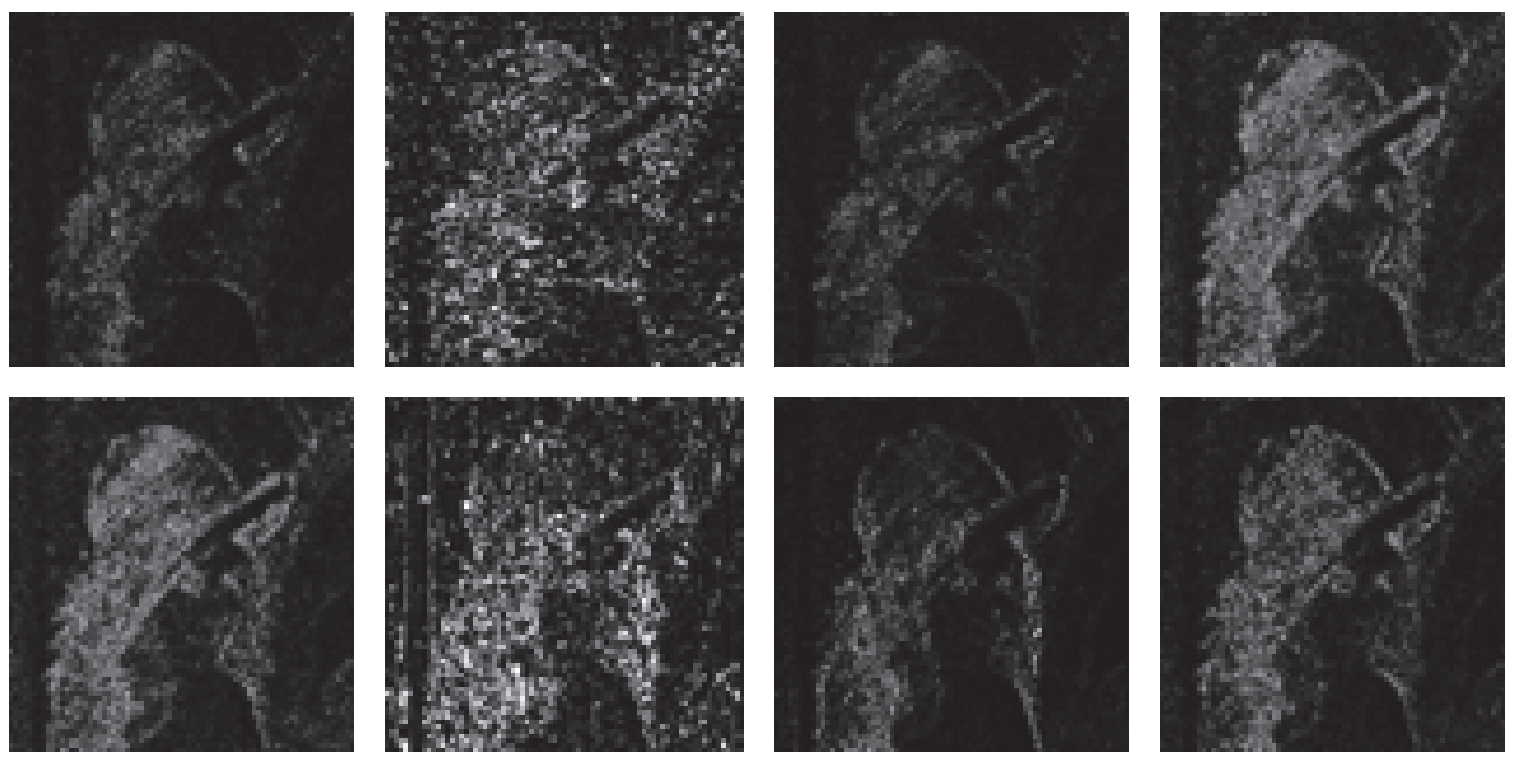

(b)

FIgURE 4: (a) is Lena. (b) Eight Shearlet direction coefficients in the time domain.

where $W$ and $W^{\prime}$ are the original and extracted watermark signals, respectively.

Figure 6 shows the extracted watermark from original images before attacking and it turns out that the watermark can be perfectly extracted.

In the following subsection,, various attacks, like JPEG compression, adding noise, median filtering et al., are performed on the original images to test the robustness of the proposed scheme.

4.1. Robustness to JPEG Compression. JPEG compression is one of the common attacks in image processing realm. Table 3 shows the values of SSIM and NC after JPEG compressing with various quality factors. It reveals that no matter what compressing factor is, NC values are all above 0.96 . Therefore, the proposed algorithm is very robust against jpeg compressing. And three extracted watermarks are selected to show visual quality in Figure 7 with the compressing factor $Q=30$. It is not hard for anyone to notice that the watermarks can be perfectly extracted.

4.2. Robustness to Median Filtering. Figure 8 gives the results of extracted watermarks after median filtering. From the figures, it can be concluded that the performance of the proposed algorithm in anti-median filtering is different among different host images. For example, the SSIM values of the host image Baboon show that the image is easily distorted by median filtering. And thus, it cannot get strong robustness like other host images. Table 4 shows the SSIM and NC values after three kinds of median filtering. And three extracted watermarks are selected to show visual quality in Figure 8 after $5 \times 5$ median filtering.

4.3. Robustness to Gaussian Noise and Salt and Pepper Noise. Adding noise is carried out to test antiattacking capacity with different standard deviations. Tables 5 and 6 show SSIM values of distorted host images and $\mathrm{NC}$ values under different variances of Gaussian noise and Salt and Pepper noise when the noise means are all set to 0 . And the standard deviations are set at $0.001,0.005,0.01,0.05$, and 0.1 . Figures 9 and 10 show the quantitative results of extracted watermarks. From the figures, though noise brings perceptibility distortion to the host images, the extracted watermarks can be seen clearly.

4.4. Robustness to Scaling. Four scaling operations are utilized to deteriorate the host images and scaling parameters 


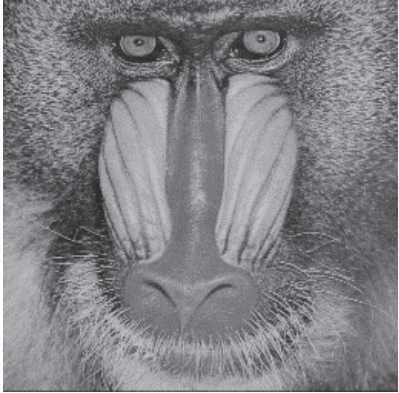

(a)

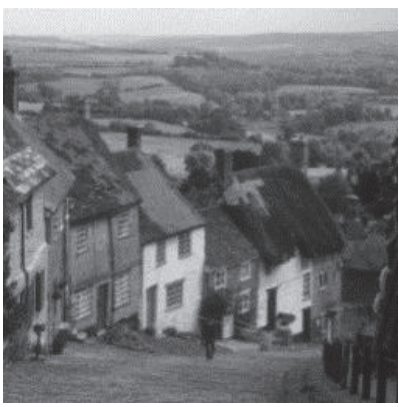

(d)

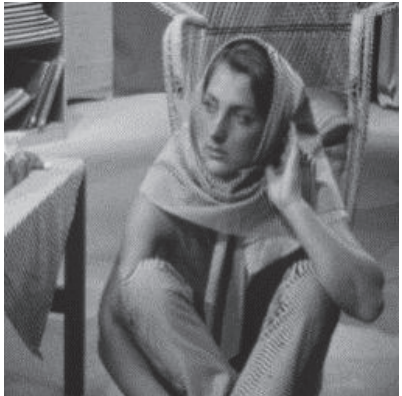

(b)

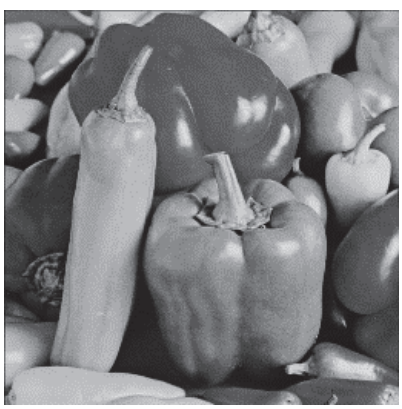

(e)

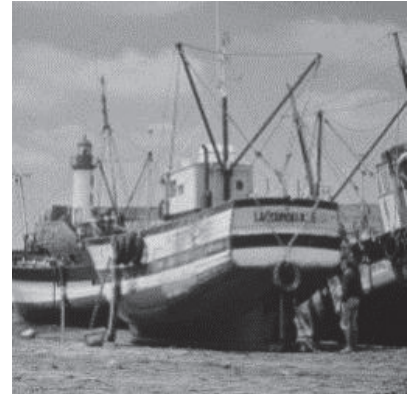

(c)

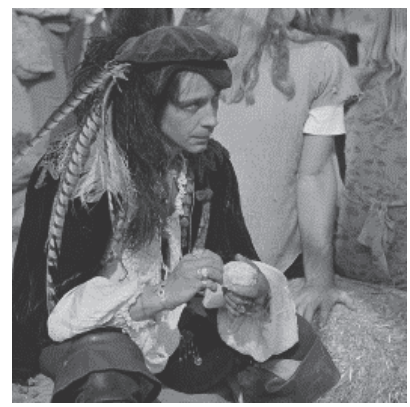

(f)

Figure 5: The host images (a) Baboon, (b) Barbara, (c) Boats, (d) Goldhill, (e) Peppers, and (f) Man.

TABLE 3: The SSIM values of distorted host images and NC values of extracted watermarks after JPEG compressing.

\begin{tabular}{lccccc}
\hline Host image & JPEG20 & JPEG30 & JPEG40 & JPEG60 & JPEG70 \\
SSIM/NC & SSIM/NC & $0.98 / 0.9948$ & $0.99 / 0.9980$ \\
\hline Boat & $0.90 / 0.9961$ & $0.93 / 0.9987$ & $0.94 / 0.9980$ & $0.97 / 0.9961$ & $0.99 / 0.9980$ \\
Goldhill & $0.84 / 0.9830$ & $0.89 / 0.9869$ & $0.90 / 0.9882$ & $0.89 / 0.9889$ & $0.92 / 0.9830$ \\
Baboon & $0.78 / 0.9671$ & $0.83 / 0.9830$ & $0.85 / 0.9829$ & $0.98 / 0.9993$ & $0.99 / 1.0000$ \\
Barbara & $0.87 / 0.9974$ & $0.91 / 0.9980$ & $0.93 / 0.9974$ & $0.89 / 1.0000$ & $0.90 / 1.0000$ \\
Peppers & $0.83 / 0.9993$ & $0.86 / 0.9993$ & $0.87 / 0.9987$ & $0.92 / 0.9954$ & $0.94 / 0.9948$ \\
Man & $0.84 / 0.9882$ & $0.88 / 0.9928$ & $0.90 / 0.9935$ & & \\
\hline
\end{tabular}

\section{A H B G}

FIGURE 6: The extracted watermark from all host images before attacking $(\mathrm{NC}=1.0000)$.

are $1 / 2,1 / 4,2$, and 4 . Table 7 shows the values of SSIM and $\mathrm{NC}$ at different scaling parameters. And Figure 11 shows the attacked images and extracted watermarks at scaling parameter $=1 / 4$. From the SSIM values, it shows that the scaling attack brings little distortion to host images except that when scaling parameter is $1 / 2$. But the extracted watermark is still pretty similar to the original watermark according to $\mathrm{NC}$ values.

4.5. Robustness to Rotating. No matter images are rotated by which angle, the inherent image direction information cannot be altered. In conventional DWT based algorithms, images are only decomposed in horizontal, vertical, or diagonal direction. Direction shortness in DWT leads to poor performance in analyzing rotated images. However, this kind of disadvantage can be perfectly resolved by NSST. Both transform directions and bases structure can be adjusted to adapt rotated images. Table 8 shows SSIM and NC values in experiments of six images rotated in 5 kinds of angles. And from Figure 12, the watermarks can be extracted without too much distortion.

4.6. The Comparison of Related Algorithm. Comparative experiments are implemented in order to verify the antiattacking capacity between the proposed algorithm in this paper and the other two schemes in paper [5], which use the counterpart transform, DWT. The scheme-1 first divide the host image into overlapping blocks. Each block is further decomposed up to level-one using DWT followed by singular value decomposition. Scheme-2 first applies DWT on the 

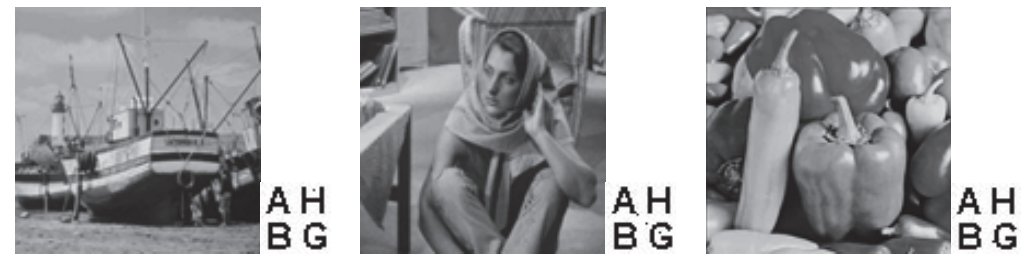

FIGURE 7: The attacked host images and extracted watermarks from Boats, Barbara, and Peppers after JPEG compressing $(Q=30)$.
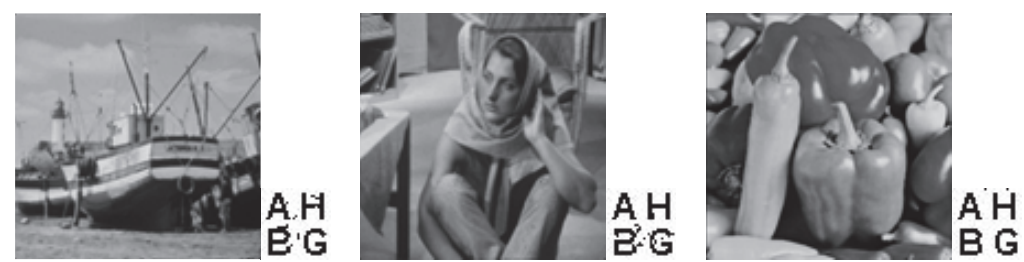

FIgURE 8: The attacked host images and extracted watermarks from Boats, Barbara, and Peppers after $5 \times 5$ median filtering.
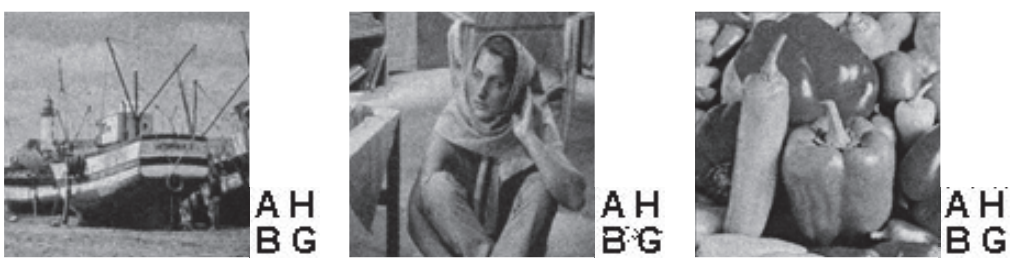

FIGURE 9: The attacked host images and extracted watermarks from Boats, Barbara, and Peppers under Gaussian noise $(v=0.01)$.
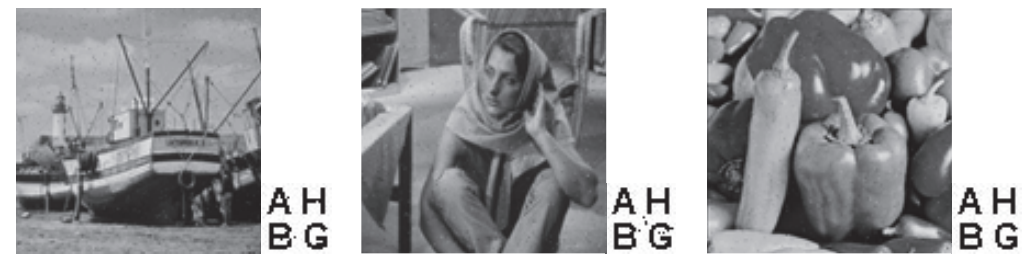

Figure 10: The attacked host images and extracted watermarks from Boats, Barbara, and Peppers under Salt and Pepper noise $(v=0.01)$.
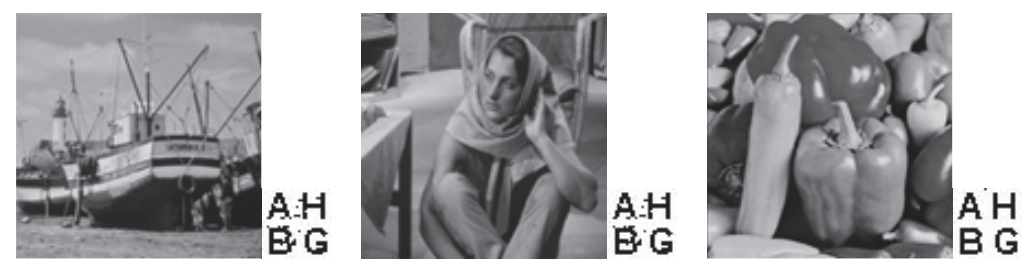

Figure 11: The attacked host images and extracted watermarks from Boats, Barbara, and Peppers after scaling (1/4).

TABLE 4: The SSIM values of distorted host images and NC values of extracted watermarks after median filtering.

\begin{tabular}{lccc}
\hline Host image & $\begin{array}{c}\text { Median filter } 3 \times 3 \\
\text { SSIM/NC }\end{array}$ & $\begin{array}{c}\text { Median filter } 5 \times 5 \\
\text { SSIM/NC }\end{array}$ & $\begin{array}{c}\text { Median filter } 7 \times 7 \\
\text { SSIM/NC }\end{array}$ \\
\hline Boat & $0.93 / 0.9823$ & $0.83 / 0.9862$ & $0.75 / 0.9849$ \\
Goldhill & $0.89 / 0.9744$ & $0.77 / 0.9711$ & $0.69 / 0.9697$ \\
Baboon & $0.73 / 0.9596$ & $0.51 / 0.9515$ & $0.42 / 0.9576$ \\
Barbara & $0.84 / 0.9836$ & $0.67 / 0.9830$ & $0.65 / 0.9643$ \\
Peppers & $0.89 / 0.9993$ & $0.85 / 0.9987$ & $0.83 / 0.9987$ \\
Man & $0.90 / 0.9796$ & $0.80 / 0.9750$ & $0.73 / 0.9710$ \\
\hline
\end{tabular}


TABLE 5: The SSIM values of distorted host images and NC values of extracted watermarks under Gaussian noise.

\begin{tabular}{lccccc}
\hline Host image & $v=0.001$ & $v=0.005$ & $v=0.01$ & $v=0.05$ & $v=0.1$ \\
SSIM/NC & SSIM/NC & SSIM/NC & $0.13 / 0.8728$ & $0.08 / 0.6977$ \\
\hline Boat & $0.72 / 1.0000$ & $0.43 / 0.9980$ & $0.32 / 0.9974$ & $0.11 / 0.8644$ & $0.07 / 0.8870$ \\
Goldhill & $0.76 / 0.9922$ & $0.45 / 0.9876$ & $0.31 / 0.9830$ & $0.20 / 0.6668$ & $0.13 / 0.7733$ \\
Baboon & $0.85 / 0.9941$ & $0.61 / 0.9719$ & $0.48 / 0.9562$ & $0.17 / 0.9915$ & $0.11 / 0.9683$ \\
Barbara & $0.77 / 0.9974$ & $0.50 / 0.9961$ & $0.39 / 0.9941$ & $0.10 / 0.9922$ & $0.06 / 0.8675$ \\
Peppers & $0.70 / 0.9987$ & $0.38 / 0.9987$ & $0.26 / 0.9993$ & $0.12 / 0.8182$ & $0.08 / 0.8577$ \\
Man & $0.75 / 0.9961$ & $0.45 / 0.9902$ & $0.33 / 0.9823$ & & \\
\hline
\end{tabular}

TABLE 6: The SSIM values of distorted host images and NC values of extracted watermarks under Salt and Pepper noise.

\begin{tabular}{lccccc}
\hline Host image & $v=0.001$ & $v=0.005$ & $v=0.01$ & $v=0.05$ & $v=0.1$ \\
& SSIM/NC & SSIM/NC & SSIM/NC & SSIM/NC & $0.38 / 0.9922$ \\
Boat & $0.97 / 1.0000$ & $0.88 / 0.9993$ & $0.78 / 0.9961$ & $0.38 / 0.9723$ & $0.21 / 0.9393$ \\
Goldhill & $0.98 / 0.9993$ & $0.89 / 0.9967$ & $0.79 / 0.9889$ & $0.50 / 0.7856$ & $0.32 / 0.8135$ \\
Baboon & $0.98 / 0.9961$ & $0.91 / 0.9711$ & $0.85 / 0.8992$ & $0.43 / 0.9928$ & $0.26 / 0.9928$ \\
Barbara & $0.98 / 0.9993$ & $0.90 / 0.9993$ & $0.81 / 0.9980$ & $0.33 / 0.9830$ & $0.17 / 0.9381$ \\
Peppers & $0.97 / 1.0000$ & $0.87 / 0.9993$ & $0.76 / 0.9993$ & $0.38 / 0.8949$ & $0.21 / 0.8662$ \\
Man & $0.98 / 0.9967$ & $0.89 / 0.9830$ & $0.79 / 0.9777$ & & \\
\hline
\end{tabular}

TABLE 7: The SSIM values of distorted host images and NC values of extracted watermarks after scaling.

\begin{tabular}{lcccc}
\hline Host image & $1 / 2$ & $1 / 4$ & 2 & 4 \\
SSIM/NC & SSIM/NC & SSIM/NC & $0.99 / 0.9980$ & $0.99 / 0.9980$ \\
\hline Boat & $0.91 / 0.9836$ & $0.97 / 0.9810$ & $0.99 / 0.9915$ & $0.99 / 0.9915$ \\
Goldhill & $0.88 / 0.9763$ & $0.96 / 0.9869$ & $0.97 / 0.9928$ & $0.97 / 0.9922$ \\
Baboon & $0.73 / 0.9676$ & $0.88 / 0.9770$ & $0.98 / 0.9922$ & $0.98 / 0.9922$ \\
Barbara & $0.80 / 0.9783$ & $0.92 / 0.9889$ & $0.98 / 1.0000$ & $0.98 / 1.0000$ \\
Peppers & $0.89 / 0.9817$ & $0.93 / 0.9993$ & $0.99 / 0.9948$ & $0.99 / 0.9948$ \\
Man & $0.89 / 0.9784$ & $0.95 / 0.9856$ & \\
\hline
\end{tabular}

TABLE 8: The SSIM values of distorted host images and NC values of extracted watermarks after rotating.

\begin{tabular}{|c|c|c|c|c|c|}
\hline Host image & $\begin{array}{c}5^{\circ} \\
\text { SSIM/NC }\end{array}$ & $\begin{array}{c}10^{\circ} \\
\text { SSIM/NC }\end{array}$ & $\begin{array}{c}15^{\circ} \\
\text { SSIM/NC }\end{array}$ & $\begin{array}{c}20^{\circ} \\
\text { SSIM/NC }\end{array}$ & $\begin{array}{c}40^{\circ} \\
\text { SSIM/NC }\end{array}$ \\
\hline Boat & $0.87 / 0.9776$ & $0.83 / 0.9776$ & $0.81 / 0.9716$ & $0.79 / 0.9763$ & $0.75 / 0.9706$ \\
\hline Goldhill & $0.83 / 0.9643$ & $0.80 / 0.9629$ & $0.77 / 0.9616$ & $0.75 / 0.9677$ & $0.71 / 0.9669$ \\
\hline Baboon & $0.66 / 0.9389$ & $0.64 / 0.9327$ & $0.62 / 0.9327$ & $0.61 / 0.9343$ & $0.58 / 0 / 9334$ \\
\hline Barbara & $0.75 / 0.9608$ & $0.72 / 0.9622$ & $0.69 / 0.9662$ & $0.67 / 0.9673$ & $0.63 / 0.9673$ \\
\hline Peppers & $0.85 / 0.9809$ & $0.82 / 0.9776$ & $0.79 / 0.9762$ & $0.77 / 0.9885$ & $0.73 / 0.9849$ \\
\hline Man & $0.83 / 0.9466$ & $0.81 / 0.9466$ & $0.78 / 0.9418$ & $0.76 / 0.9549$ & $0.72 / 0.9590$ \\
\hline
\end{tabular}
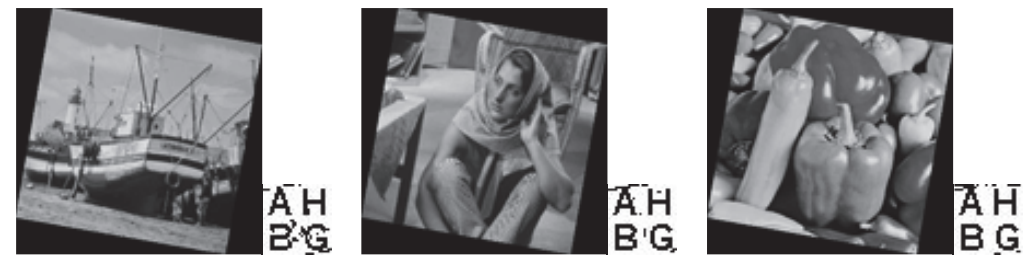

FIGURE 12: The attacked host images and extracted watermarks from Boats, Barbara, and Peppers after rotating $\left(10^{\circ}\right)$. 
TABLE 9: Seven kinds of attacking style.

\begin{tabular}{lc}
\hline & Attacking style \\
\hline 1 & Gaussian noise adding $(5 \%)$ \\
2 & JPEG compression $(Q=40)$ \\
3 & Resizing $(512-256-512)$ \\
4 & Median filtering $(5 \times 5)$ \\
5 & Average filtering $(7 \times 7)$ \\
6 & Rotate \\
7 & Histogram Equalization \\
\hline
\end{tabular}

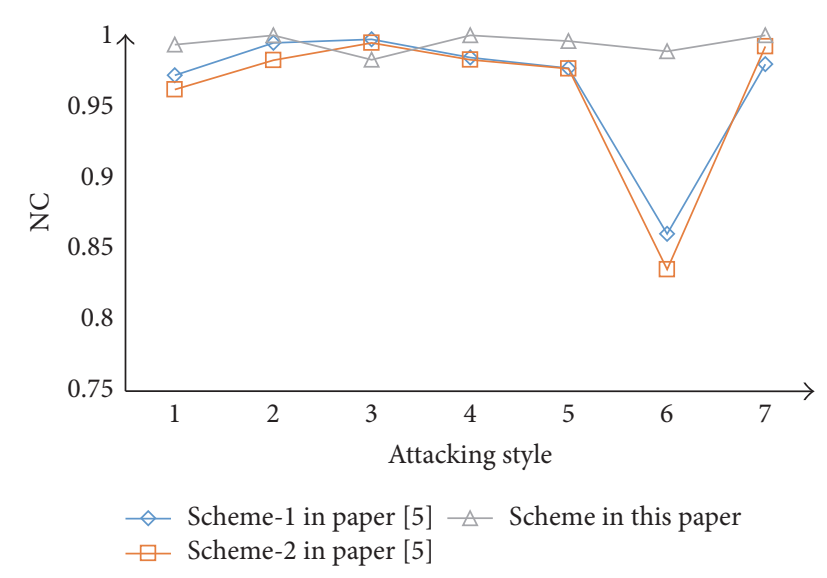

FIGURE 13: Similarity comparison statistics of watermark extracted from Peppers.

whole image and LL subband is then selected for the following processing.

Figures 13 and 14 demonstrate NC values comparing results and seven kinds of attacking style are listed in Table 9. The host images are Peppers and Barbara, respectively. It can be easily seen from these two figures that the proposed scheme in this paper generally outperforms the other two zero-watermarking methods. From Figure 13, the antiattacking ability of the proposed scheme is higher than other two methods, except that NC value of the third attacking is a bit lower. From Figure 14, NC values of our scheme are lower than the scheme-1 when attacking is resizing (512-256-512), median filtering $(5 \times 5)$, and average filtering $(7 \times 7)$. But according to the experimental data, NC values of our scheme are all above 0.96 . That is to say, the proposed scheme is also a good method.

However, it cannot be ignored that DWT based methods are slightly better than the proposed algorithm in antiresizing. DWT has been widely used in watermarking mainly owing to its good ability in modeling the human visual system. The most important feature of NSST is its appropriate representation of direction feature parts, like textures or edges. When images are resized from $512 \times 512$ to $256 \times 256$, some direction feature information may be missing to some extent. This is the main reason why extracted watermark is slightly different from the original. However, it cannot be overlooked that the proposed algorithm shows the strong

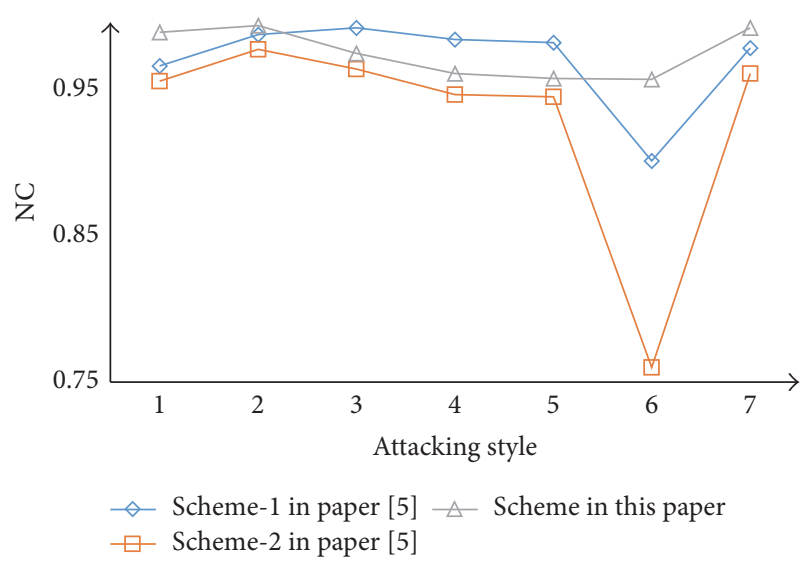

FIGURE 14: Similarity comparison statistics of watermark extracted from Barbara.

robustness ability significantly against rotating than the other two methods.

From the above analysis, the proposed algorithm takes more advantage in robustness performance and, therefore, it gets a higher security than the other two zero-watermarking algorithms.

\section{Conclusions}

A digital watermarking scheme based on NSST has been described in this paper. Experimental results demonstrate that the NSST improves the digital watermarking performance considerably when compared to other methods based on DWT. The results show the good robustness against common attacks. In conclusion, it can be argued that a NSST-based watermarking scheme has a good perspective for effective digital right management.

\section{Competing Interests}

The authors declare that they have no competing interests.

\section{Acknowledgments}

This work was supported by Scientific Research Starting Foundation of Northwest University, National Natural Science Foundation of China (nos. 61379010 and 61572400), and Natural Science Basic Research Plan in Shaanxi Province of China (no. 2015JM6293).

\section{References}

[1] B. Ahmederahgi, F. Kurugollu, P. Milligan, and A. Bouridane, "Spread spectrum image watermarking based on the discrete shearlet transform," in Proceedings of the 2013 4th European Workshop on Visual Information Processing (EUVIP '13), pp. 178-183, Paris, France, June 2013.

[2] A. Rani and B. Raman, "An image copyright protection scheme by encrypting secret data with the host image," Multimedia Tools and Applications, vol. 75, no. 2, pp. 1027-1042, 2016. 
[3] W.-H. Lin, Y.-R. Wang, and S.-J. Horng, "A wavelet-tree-based watermarking method using distance vector of binary cluster," Expert Systems with Applications, vol. 36, no. 6, pp. 9869-9878, 2009.

[4] W.-H. Lin, S.-J. Horng, T.-W. Kao et al., "Image copyright protection with forward error correction," Expert Systems with Applications, vol. 36, no. 9, pp. 11888-11894, 2009.

[5] A. Rani, A. K. Bhullar, D. Dangwal, and S. Kumar, "A zerowatermarking scheme using discrete wavelet transform," Procedia Computer Science, vol. 70, pp. 603-609, 2015.

[6] G. Kutyniok, W.-Q. Lim, and G. Steidl, "Shearlets: theory and applications," GAMM-Mitteilungen, vol. 37, no. 2, pp. 259-280, 2014.

[7] G. Bhatnagar, Q. M. J. Wu, and P. K. Atrey, "Secure randomized image watermarking based on singular value decomposition," ACM Transactions on Multimedia Computing, Communications and Applications, vol. 10, no. 1, article 4, 2013.

[8] G. Liu, H. Liu, and A. Kadir, "Wavelet-based color pathological image watermark through dynamically adjusting the embedding intensity," Computational and Mathematical Methods in Medicine, vol. 2012, Article ID 406349, 10 pages, 2012.

[9] R. H. Bamberger and M. J. T. Smith, "A filter bank for the directional decomposition of images: theory and design," IEEE Transactions on Signal Processing, vol. 40, no. 4, pp. 882-893, 1992.

[10] A. L. Da Cunha, J. Zhou, and M. N. Do, “The nonsubsampled contourlet transform: theory, design, and applications," IEEE Transactions on Image Processing, vol. 15, no. 10, pp. 3089-3101, 2006.

[11] C. Qin and X. Wen, "A novel digital watermarking algorithm in contourlet domain," Journal of Information \& Computational Science, vol. 11, no. 2, pp. 519-526, 2014.

[12] H. Song, S. Yu, X. Yang, L. Song, and C. Wang, "Contourletbased image adaptive watermarking," Signal Processing: Image Communication, vol. 23, no. 3, pp. 162-178, 2008.

[13] G. Easley, D. Labate, and W.-Q. Lim, "Sparse directional image representations using the discrete shearlet transform," Applied and Computational Harmonic Analysis, vol. 25, no. 1, pp. 25-46, 2008.

[14] G. R. Easley, D. Labate, and W. Q. Lim, "Optimally sparse image representations using shearlets," in Proceedings of the Fortieth Asilomar Conference on Signals, Systems and Computers (ACSSC '06), pp. 974-978, Pacific Grove, Calif, USA, November 2006.

[15] D. Labate, W.-Q. Lim, G. Kutyniok, and G. Weiss, "Sparse multidimensional representation using shearlets," in Proceedings of the Curvelet, Directional, and Sparse Representations I, vol. 5914 of Proceedings of SPIE, pp. 254-262, San Diego, Calif, USA, July 2005.

[16] Q. Wen, T.-F. Sun, and S.-X. Wang, "Concept and application of zero-watermark," Acta Electronica Sinica, vol. 31, no. 2, pp. 214216, 2003.

[17] S.-C. Han and Z.-N. Zhang, "A novel zero-watermark algorithm based on LU decomposition in NSST domain," in Proceedings of the 11th International Conference on Signal Processing (ICSP '12), pp. 1592-1596, Beijing, China, October 2012.

[18] Z. Jian, S. Meiling, J. Jian, H. Luxi, H. Fan, and L. Shan, "Image watermark based on extended shearlet and insertion using the largest information entropy on horizontal cone," Mathematical Problems in Engineering, vol. 2015, Article ID 450819, 10 pages, 2015.
[19] W.-Q. Lim, "The discrete shearlet transform: a new directional transform and compactly supported shearlet frames," IEEE Transactions on Image Processing, vol. 19, no. 5, pp. 1166-1180, 2010.

[20] C. Bergman and J. Davidson, "Unitary embedding for data hiding with the SVD," in Proceedings of the Security, Steganography, and Watermarking of Multimedia Contents VII, vol. 5681 of Proceedings of SPIE, San Jose, Calif, USA, January 2004.

[21] B. Zhou and J. Chen, "A geometric distortion resilient image watermarking algorithm based on SVD," Journal of Image and Graphics, vol. 4, article 022, 2004.

[22] X.-J. He, Y. Zhang, T.-M. Lok, and M. R. Lyu, "A new feature of uniformity of image texture directions coinciding with the human eyes perception," in Proceedings of the 2nd International Conference on Fuzzy Systems and Knowledge Discovery (FSKD '05), pp. 727-730, Changsha, China, August 2005.

[23] T. Kubo, M. Obuchi, G. Ohashi, and Y. Shimodaira, "Image processing system for direction detection of an object using neural network," in Proceedings of the IEEE Asia-Pacific Conference on Circuits and Systems (IEEE APCCAS '98), pp. 571-574, Chiangmai, Thailand, December 1998.

[24] Z. Li, H. He, H.-M. Tai, Z. Yin, and F. Chen, "Color-direction patch-sparsity-based image inpainting using multidirection features," IEEE Transactions on Image Processing, vol. 24, no. 3, pp. 1138-1152, 2015.

[25] Z. Wang, A. C. Bovik, H. R. Sheikh, and E. P. Simoncelli, "Image quality assessment: from error visibility to structural similarity," IEEE Transactions on Image Processing, vol. 13, no. 4, pp. 600612, 2004. 


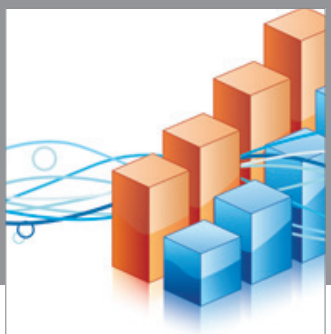

Advances in

Operations Research

vatem alat4

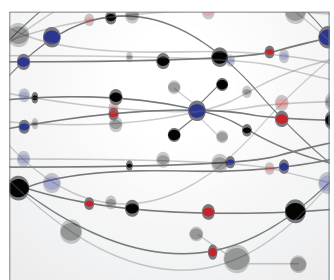

\section{The Scientific} World Journal
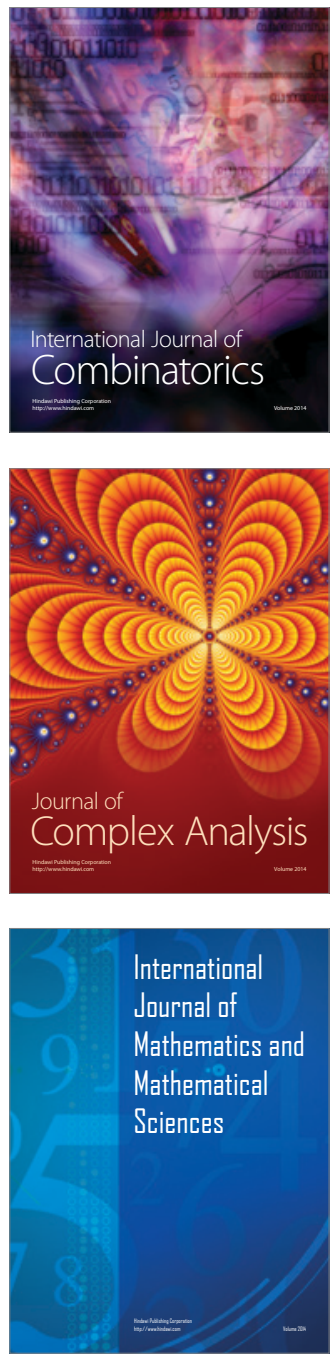
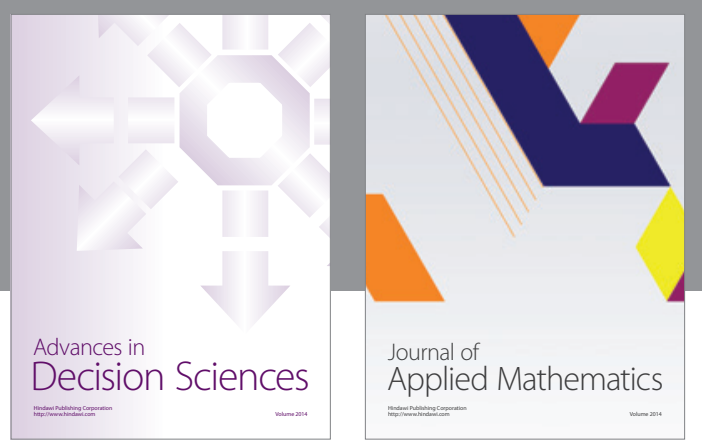

Algebra

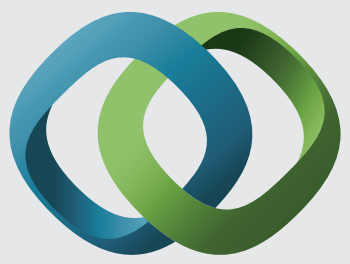

\section{Hindawi}

Submit your manuscripts at

http://www.hindawi.com
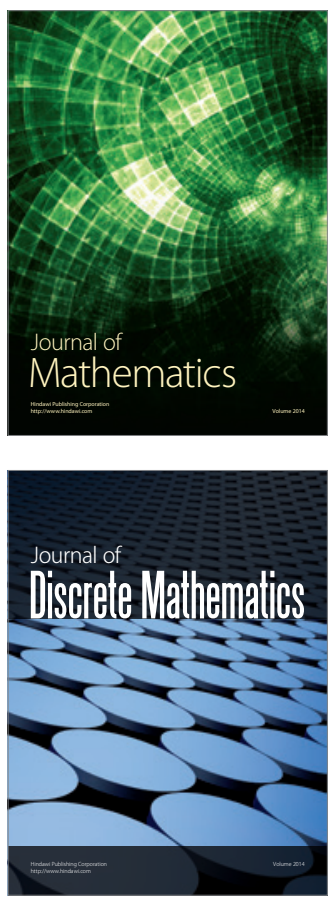

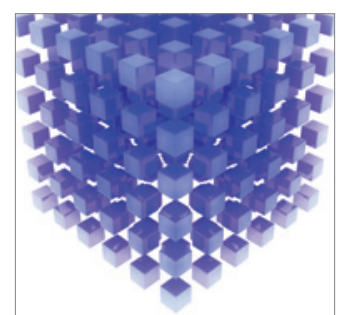

Mathematical Problems in Engineering
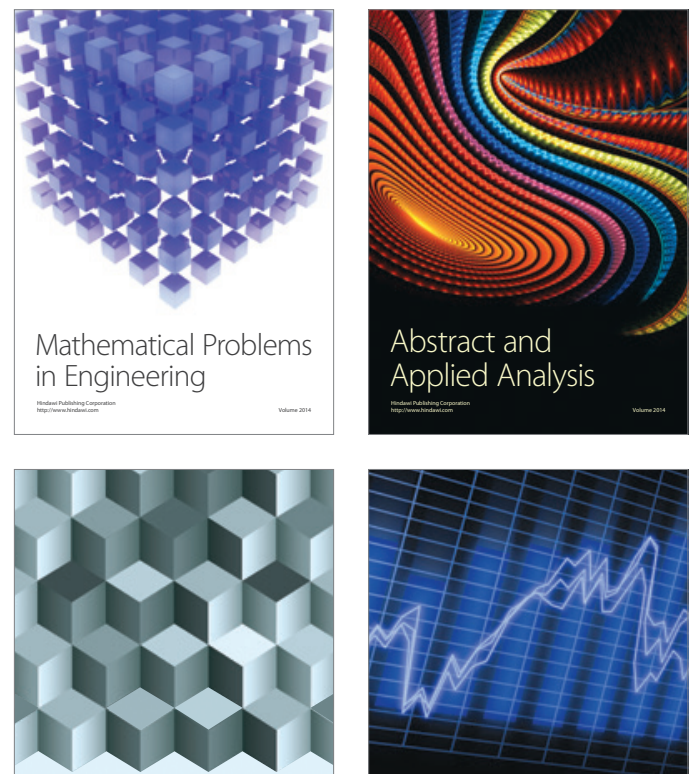

Journal of

Function Spaces

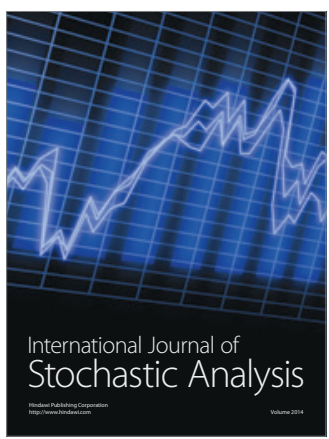

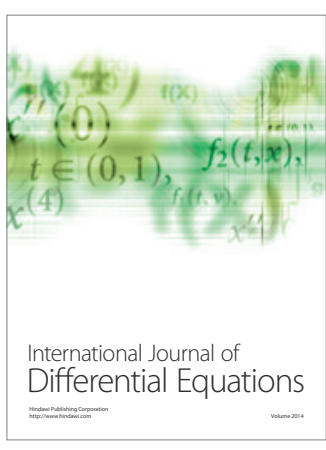
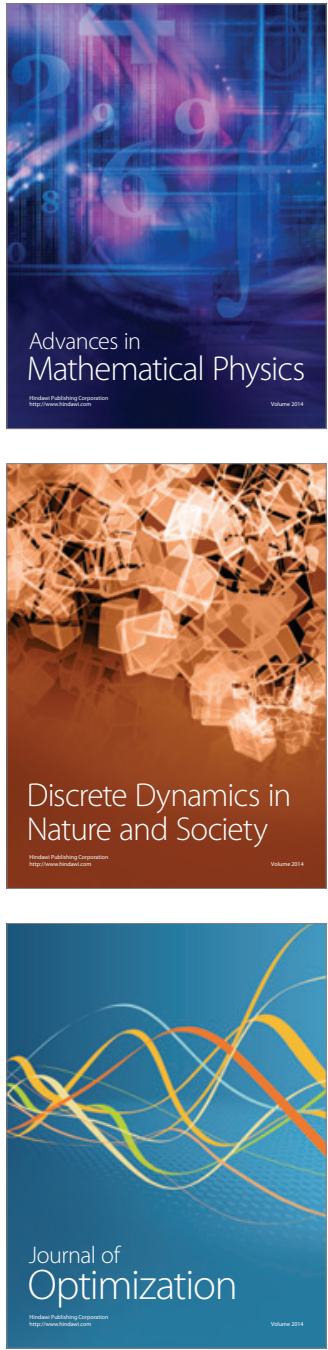